\title{
Orientación estratégica y desempeño de negocios artesanales afectados por crisis en Oaxaca, México*
}

\author{
Mendoza Ramírez, Luis** \\ Toledo López, Arcelia***
}

\section{Resumen}

La literatura sobre emprendedurismo y gestión estratégica sugiere el efecto positivo de la orientación estratégica (OE) sobre el desempeño de las empresas en ambientes hostiles; no obstante, los resultados son de empresas grandes de países desarrollados; en circunstancias de estabilidad económica y social, donde el ambiente hostil se debe a factores tecnológicos, industriales o de mercado. El objetivo de este estudio es analizar el desempeño de negocios artesanales como resultado de la OE de los dueños, en un marco de crisis de deterioro económico, inestabilidad social y cambio climático en Oaxaca, México. Con base en los datos de 101 negocios artesanales, analizados con correlación bivariada de Pearson y regresión lineal múltiple, los resultados del estudio indican un efecto positivo de innovación y proactividad sobre el desempeño. En contra de lo esperado, se encuentra un efecto negativo de agresividad competitiva sobre el desempeño. Se concluye que, aunque los dueños de negocios artesanales se comprometen en tres dimensiones de OE para mejorar el desempeño, no todas ellas tienen efecto, ni logran modificarlo de la misma manera, debido a las condiciones contextuales.

Palabras clave: Pequeños negocios, desempeño del negocio, orientación estratégica, crisis, México.

\section{Recibido: 10-05-12. Aceptado: 04-11-13}

* Los resultados de este estudio corresponden a resultados parciales de un trabajo de investigación de mayor alcance, que incluye otras variables y que en conjunto conformaran los resultados de una tesis doctoral en curso.

** Contador Público, Universidad Autónoma Benito Juárez de Oxaca (UABJO), México; Maestro en Ciencias, Instituto Politécnico Nacional (IPN), Centro Interdisciplinario de Investigación para el Desarrollo Integral Regional (CIIDIR), Oaxaca, México; Doctorando del IPN, CIIDIR, e-mail: coatlihuec@hotmail.com

*** Profesor del Instituto Politécnico Nacional. IPN CIIDIR-Oaxaca, México, e-mail: artoledol@ ipn.mx 


\title{
Strategic Orientation and Performance of Small Businesses in a Crisis Environment
}

\begin{abstract}
Literature on entrepreneurship and strategic management suggests the positive effect of strategic orientation on company performance in hostile environments; nevertheless, these results are based on large companies from developed countries, under circumstances of economic and social stability, where the hostile environment is due to technological, industrial or market factors. The aim of this study is to analyze the performance of small handicraft businesses as a result of the owners' strategic orientation in a context of crisis caused by economic decline, social instability and climate change in Oaxaca, Mexico. Pearson bivariate correlation and multiple linear regressions are used to analyze data from 101 handicraft businesses. Results indicate the positive effect of innovation and proactivity on performance. Contrary to expectations, a negative effect of competitive aggressiveness on performance was found. Conclusions are that, although the craft business owners engage in three dimensions of strategic orientation to improve performance, not all of them have an effect, nor do they modify performance in the same way, due to contextual conditions.
\end{abstract}

Key words: Small businesses, business performance, strategic orientation, crisis, Mexico.

\section{Introducción}

La literatura sobre emprendedurismo y gestión estratégica intenta explicar el desempeño de las empresas con base en su postura estratégica (Lumpkin y Dess, 1996; Covin y Slevin, 1989; Venkatraman, 1989). En general, los estudios sugieren un efecto positivo de OE sobre el desempeño, en empresas de diferente industria, tamaño y propiedad (Rosenbusch et al., 2013; Bamiatzi y Kirchmaier, 2012; Rauch et al., 2009; Wang y Zhang, 2009; Escribá-Esteve et al., 2008; Huasiu, 2006; Ripollés y Blesa, 2006). Una parte importante de esos estudios muestra evidencia de tal efecto en el desempeño de empresas inmersas en ambientes hostiles (Martins y Rialp, 2013; Rosenbusch et al., 2013; Wales et al., 2013; Kraus et al., 2012; Naidoo, 2010; Lumpkin y Dess, 2001; Covin y Slevin, 1989; Hall, 1980).
La evidencia aportada en esos estudios se basa en investigaciones realizadas en empresas formalmente establecidas de países desarrollados; en circunstancias de estabilidad económica y social, donde el ambiente hostil se debe a factores tecnológicos, industriales, legales o de mercado. Sin embargo, hasta donde se sabe, existen muy pocos estudios que proporcionan indicios del efecto de la OE o de algunas de sus dimensiones, sobre el desempeño de los negocios sin una estructura formal, en el contexto de economías no desarrolladas (Domínguez et al., 2008; Hernández et al., 2010). No obstante, esos estudios no consideran el ambiente en que se desenvuelven los negocios analizados, o analizan el desempeño desde una perspectiva de la estrategia diferente a la OE.

Con base en estas consideraciones y teniendo en cuenta que el contexto de un país desarrollado es diferente al de 
un país en desarrollo y que, como consecuencia también las empresas en ambos contextos son diferentes debido al ambiente en que operan (Lumpkin et al., 2013; Anderson y Eshima, 2011); el modelo de OE utilizado en estudios realizados en países desarrollados puede no tener los mismos resultados en empresas de países con diferente grado de desarrollo. Por lo tanto, el presente trabajo tiene como objetivo analizar el desempeño de los pequeños negocios artesanales, como resultado de la OE de los dueños, en un marco de contingencia originada por deterioro económico, inestabilidad social y cambio climático en Oaxaca, México.

De esa manera se trata de contribuir en la literatura existente para tener una mejor comprensión del efecto de la OE en sus dimensiones de innovación, agresividad competitiva y proactividad, para explicar el desempeño de las empresas afectadas por ambientes hostiles de crisis económica, social y de la naturaleza.

Para ello, el estudio se lleva a cabo en un contexto de pequeños negocios de una economía en desarrollo, donde éstos juegan un papel muy importante por su contribución al bienestar de las comunidades en que operan. Tal es el caso de los negocios de artesanía en Oaxaca, México; que contribuyen económicamente al atraer turismo nacional e internacional generando empleos directos e indirectos; además de apoyar a la infraestructura pública y la conservación de las celebraciones religiosas y culturales de sus comunidades (Toledo-López et al., 2012; Toledo et al., 2010), pero que también son los que más sufren los efectos del ambiente hostil (Hernández et al., 2010).
La selección de esos negocios para realizar el estudio obedece a la situación de crisis en que operan y que impacta en su desempeño debido a tres factores: las secuelas de la crisis financiera mundial; las consecuencias del conflicto políticosocial de 2006, a las que se suma la inseguridad pública derivada de la violencia desatada por la lucha contra el crimen en México, y los eventos de la naturaleza provocados por el cambio climático.

Tal situación de crisis plantea un marco de contingencia que impone a los dueños de negocio el reto por sobrevivir mediante un replanteamiento de las estrategias, adoptando una postura que les ayude a sobrellevar exitosamente el ambiente incierto en que operan; y la exigencia de mejora y aprovechamiento de las eficiencias internas del negocio, enfocándose en el producto y el proceso con el objetivo de mejorar el desempeño.

Dado que las crisis por definición son sucesos impredecibles, la situación de contingencia en que operan los negocios de artesanía en Oaxaca presenta una excelente oportunidad para analizar el efecto de la OE sobre el desempeño, en un ambiente hostil representado por crisis económica, social y de la naturaleza.

La metodología utilizada fue cuantitativa, de tipo transversal y se basó en datos obtenidos en una encuesta aplicada a 101 propietarios (dueños) de pequeños negocios de cuatro ramas artesanales: alfarería (39), textil (32), tallado en madera (16) y metalistería (14); ubicados en la región de los Valles Centrales y Sierra Sur de Oaxaca. La antigüedad promedio de los negocios era de 23 años. De los encuestados, $60 \%$ son hombres y $40 \%$ son mujeres; cuya edad promedio fue 45 
Orientación estratégica y desempeño de negocios artesanales afectados por crisis... Mendoza Ramírez, Luis y Toledo López, Arcelia

años. La colección de datos se llevó a cabo de marzo a mayo de 2011, mediante un cuestionario estructurado con un enfoque temporal en referencia a cada uno de los periodos de crisis.

La validez interna de las variables del estudio se determinó mediante un análisis factorial, utilizando el método de extracción de componentes principales con rotación Varimax con Kaiser. La fiabilidad se calculó mediante el coeficiente del Alfa de Cronbach. La evaluación del modelo conceptual se realizó mediante análisis de correlación bivariada de Pearson y regresión múltiple.

Se pretende describir el contexto de los negocios de artesanía en Oaxaca, el ambiente de crisis en que operan y su impacto en el desempeño; para ello parte de la revisión de la teoría relacionada al tema de estudio, de donde se desprende el modelo de investigación.

\section{Los negocios de artesanía en Oaxaca}

Los negocios de artesanía en Oaxaca generalmente son pequeñas unidades de producción, carentes de una estructura organizacional formal en donde todos los miembros de la familia participan en las actividades del negocio (Hernández et al., 2010), por lo que la mayoría no contrata empleados para llevar a cabo las diferentes etapas del proceso de producción. Debido a esto, por lo general carecen de registros e indicadores para evaluar la actuación de los empleados en los resultados financieros del negocio; que por otra parte, son difíciles de cono- cer; debido a que en estos negocios los gastos de operación y los ingresos por ventas se administran de manera conjunta con el gasto doméstico. De manera que no cuentan con una contabilidad formal, por lo que poco se sabe sobre la rentabilidad real y las operaciones financieras del negocio.

La practica más común de estos negocios es la creatividad, realizan innovación mediante la adopción de nuevos materiales y equipo utilizados en el proceso de elaboración (Hernández et al., 2005; Stephen, 2005; Cook, 1995), así como la implementación de nuevos diseños, tamaños y formas en el producto (Hernández et al., 2007). La mayoría de estos negocios opera en un mercado local y para aumentar las ventas y mantener o aumentar la porción de mercado, los dueños del negocio generalmente recurren a prácticas tales como la venta de los productos a precios más bajos que los competidores, o el pago a guías de turistas para que les lleven clientes; aunque esto signifique el sacrificio de una parte de sus ganancias (Domínguez et al., 2004; Cohen, 2001).

La estrategia que utilizan para competir parece basarse en acciones orientadas a limitar las oportunidades de venta de otros negocios mediante prácticas tales como: a) la colusión con guías de turistas mediante acuerdos que establecen qué tipo de turistas reunir y qué lugares visitar; b) el control del flujo de información con respecto a los cambios en los gustos de los clientes y el mercado, y c) la calumnia, mediante el descrédito de los competidores. 


\subsection{Ambiente de crisis: orígenes y afectación al desempeño de los negocios artesanales}

El ambiente plantea situaciones difíciles y complejas que conllevan a los dueños o gerentes a tomar las decisiones que aseguren la continuidad y la adaptación de la empresa, aprovechando las oportunidades y superando las amenazas (Elbanna y Child, 2007). Tales situaciones se deben a sucesos imprevisibles que representan crisis, las cuales amenazan el desempeño y la supervivencia de las organizaciones (Weick, 1988). Las crisis pueden originarse en la economía, en el contexto social o institucional (Koselleck, 2006); o en la naturaleza (inundaciones o terremotos) y pueden provocar trastornos en las organizaciones (Abdul-Akeem, 2010).

Tal es el caso del ambiente de crisis en Oaxaca; originado en parte, por tres factores principales: 1) la desaceleración en la economía de Estados Unidos en 2008, 2) el conflicto político-social en Oaxaca en 2006, y 3) los eventos de la naturaleza en 2010. Se describen brevemente precisando como contribuyen a la crisis en que operan los negocios de artesanía en Oaxaca y su impacto en el desempeño de esos negocios.

\subsection{La desaceleración en la economía de Estados Unidos en el año 2008}

Este suceso que un año después derivó en la crisis financiera mundial, con un impacto aún más devastador que la Gran Depresión de 1929 (Munir, 2010), tuvo un impacto muy significativo en México, pues provocó la disminución en los principales indicadores económicos que conforman su fuente de ingresos externos: las exportaciones de petróleo, turismo y remesas de inmigrantes en Estados Unidos (Ritchie et al., 2010).

Este hecho repercutió negativamente en la actividad económica de todo el país; particularmente en Oaxaca, donde las consecuencias de la crisis continúan afectando la economía en forma de disminución del flujo de efectivo circulante y del valor real de los salarios; lo que a su vez repercute negativamente en el poder de compra de los consumidores. Esto representa un impacto severo en el desempeño de los negocios de artesanía; debido a que con la insuficiencia de los salarios para comprar los productos básicos, se reduce el número de clientes, ya que se anteponen las necesidades básicas frente al consumo de objetos decorativos. Finalmente, esa disminución en ventas afecta el desempeño de los negocios artesanales.

Esa afectación se incrementa aún más por la reducción en el nivel de ingresos por ventas que representa la disminución en la afluencia de turistas a Oaxaca (Segura, 2009; Chabela, 2011). Debido a que los negocios de artesanía no tienen un mercado propio y sus ventas dependen principalmente del turismo (Hernández et al., 2010; Domínguez et al., 2004), la disminución en las llegadas de turistas a Oaxaca impacta de manera directa en la venta de productos artesanales.

\subsection{El conflicto político-social en Oaxaca en el año 2006}

Evento surgido en el ámbito institucional entre gobierno y sindicato, que derivó en una crisis social con un impacto 
Orientación estratégica y desempeño de negocios artesanales afectados por crisis... Mendoza Ramírez, Luis y Toledo López, Arcelia

significativo en la actividad económica y la sociedad de Oaxaca.

Desde 1980, la sección 22 del sindicato nacional de trabajadores de la educación (SNTE) realiza acciones tales como marchas, manifestaciones permanentes y ocupación de calles como medidas de presión para lograr las demandas de su pliego petitorio. Estas acciones generalmente se presentaban durante el mes de mayo y desaparecían con la negociación del pliego petitorio.

Sin embargo, en 2006 como consecuencia de la represión gubernamental, tales acciones no solo se diversificaron e intensificaron con la participación de otras organizaciones sindicales y civiles; así como diferentes sectores de la sociedad como indígenas y grupos populares; sino que además, se prolongaron durante casi todo ese año generando una crisis social que, entre otras consecuencias provocó pérdidas por miles de millones de pesos, debido a la afectación en la actividad de varios sectores económicos, entre ellos el sector artesanal (Martínez, 2008; Zafra, 2008).

La afectación de esa crisis social en el sector artesanal se reflejó en el bajo desempeño de los negocios de artesanía, evidenciado por la disminución en su rentabilidad y crecimiento como consecuencia de la reducción en el nivel de ventas de productos artesanales (Hernández et al., 2010). En la sociedad, la crisis generó un ambiente de inestabilidad que afectó de manera importante la convivencia y la seguridad, debido a desórdenes sociales como el bloqueo de calles, marchas, manifestaciones masivas y clausura de oficinas públicas (Sorroza, 2008).
La presencia sistemática de esos desórdenes, aún después del conflicto político-social, a la que se suma la inseguridad pública derivada de la violencia desatada por la lucha contra el crimen, afecta de manera negativa el desempeño de los negocios artesanales al generar condiciones poco favorables para la comercialización de artesanías (Chabela, 2011). Por ejemplo, la instalación permanente de manifestantes en el centro de la ciudad impide a los compradores el acceso a los lugares de venta de productos artesanales; los bloqueos en las calles o carreteras provocan que los envíos de artesanías no lleguen a tiempo, causando la cancelación de pedidos y la pérdida de los clientes; y el cierre de carreteras impide el paso de los compradores a las comunidades donde se elaboran y venden artesanías.

Además, la mala imagen de Oaxaca como resultado de los constantes desórdenes sociales y de la inseguridad pública, provoca que la llegada de turismo disminuya y con ello se reducen también las posibilidades de venta para los negocios artesanales.

\subsection{Los eventos de la naturaleza en el año 2010}

De igual forma, los eventos originados en la naturaleza como tsunamis y terremotos, o los provocados por el cambio climático generan crisis. La crisis en la naturaleza representa una amenaza para el desempeño de las empresas, debido a su imprevisibilidad en magnitud, duración, fecha y ubicación (Linnenluecke y Griffiths, 2010). En particular, los eventos derivados del cambio climático, como las 
sequías prolongadas y temperaturas altas, o las lluvias intensas y continuas como las provocadas por el huracán Frank en 2010 (Mejía, 2010), tienen un impacto directo en el desempeño de los negocios de artesanía en Oaxaca, debido a que la elaboración de la mayoría de los productos artesanales depende de factores climáticos, que pueden agilizar o interrumpir el proceso de producción (Domínguez et al., 2008).

Por ejemplo, en la producción de los productos de alfarería, aunque el calor acelera el secado de las piezas, una temperatura extremadamente alta en un clima seco puede provocar que las piezas se agrieten y se rompan, provocando la pérdida de materia prima. En el otro extremo, las lluvias intensas y continuas hacen que el tiempo para lograr el secado necesario de las piezas para llevar a cabo su cocción se prolongue, debido a la humedad en el ambiente; esto provoca la interrupción de la producción y con ello el retraso en la entrega de los pedidos a los clientes.

De manera similar, el efecto de las lluvias impacta también en el proceso de elaboración de las artesanías de hojalata, debido a que la humedad en el ambiente no permite que la pintura con que se decoran las piezas tenga un secado rápido. Esto provoca un acabado opaco y una afectación directa en la calidad de los productos de hojalata.

No obstante, el impacto de los eventos del cambio climático en el desempeño no solo es de manera directa. También pueden afectar el desempeño de manera indirecta a través del efecto de flujo (Abdul-Akeem, 2010). Ejemplo de ello es el deterioro de las principales vías de comunicación terrestre como conse- cuencia de las fuertes lluvias en el año 2010. Esto provocó que hubiera muy poca afluencia de turistas en Oaxaca; lo que trajo como consecuencia un nivel muy bajo de ventas en los negocios de artesanía.

Los ejemplos mencionados ilustran brevemente el efecto negativo que tienen los eventos de la naturaleza sobre el desempeño de los negocios de artesanía; dado que generan incertidumbre en las decisiones sobre el volumen de producción, riesgo de pérdida de materiales, afectación a la calidad de los productos y posibilidad de incumplimiento de pedidos de mercancía en tiempo.

Los tres factores mencionados en este apartado: el económico, el social y de la naturaleza generan un ambiente de crisis en Oaxaca, que afecta el desempeño de los negocios de artesanía. Ante esta situación se plantean las interrogantes: ¿Cómo responden los dueños de los negocios de artesanía en Oaxaca a la crisis económica, social y de la naturaleza en que operan desde 2006?; ¿Cuál es el efecto de las acciones emprendidas por los dueños, en el desempeño del negocio?, las cuales se pretenden responder en esta investigación.

\section{Orientación Estratégica como predictor del desempeño de empresas en ambientes hostiles}

Desde el enfoque de la estrategia, la actividad emprendedora tradicionalmente se caracteriza como un tipo de respuesta estratégica en un ambiente hostil (Bamiatzi y Kirchmaier, 2012; Weaver et al., 2002). Bajo esta premisa varios auto- 
Orientación estratégica y desempeño de negocios artesanales afectados por crisis... Mendoza Ramírez, Luis y Toledo López, Arcelia

res postulan que en un ambiente adverso, la adopción de una OE es un mecanismo que permite a las empresas mantener o mejorar el desempeño (Rosenbusch et al., 2013; Wales et al., 2013; Covin y Slevin, 1989).

En un estudio clásico sobre gestión estratégica de pequeñas empresas en ambientes hostiles y benignos, Covin y Slevin (1989) argumentan que en ambientes hostiles, las empresas con índices altos de OE logran niveles más altos de desempeño. Con base en este argumento, los autores postulan la OE como un elemento predictor del desempeño en empresas que operan en ambientes hostiles. Diferentes estudios posteriores soportan ese postulado al atribuir a la OE, así como al efecto independiente de las diferentes dimensiones que la integran, tales como: análisis, innovación, proactividad y agresividad competitiva, el desempeño positivo de las empresas inmersas en ambientes hostiles originados por diferentes factores (Bamiatzi y Kirchmaier, 2012; Foster, 2010; Escribá-Esteve et al., 2008; Lumpkin y Dess, 2001).

Por ejemplo, el estudio de EscribáEsteve et al. (2008) sugiere que en un ambiente de turbulencia tecnológica y de mercado, las empresas con una postura proactiva exploran el mercado en busca de oportunidades para anticiparse a los competidores, no sin antes llevar a cabo un proceso exhaustivo de toma de decisiones. Esto les permite lograr crecimiento en ventas y porción de mercado, y la lealtad de los clientes. Lumpkin y Dess (2001) indican que en un ambiente hostil, las empresas proactivas que compiten agresivamente logran mejores índices de rentabilidad y crecimiento en ventas.
Estudios empíricos más recientes señalan la efectividad de la OE, a través de sus diferentes dimensiones para mejorar el desempeño financiero y no financiero de empresas afectadas por crisis económicas (Martins y Rialp, 2013). Esos estudios muestran que la innovación, mediante la diferenciación del producto, no sólo permite la supervivencia de las empresas; sino que impacta positivamente en su crecimiento y rentabilidad (Bamiatzi y Kirchmaier, 2012; Naidoo, 2010). También proporcionan evidencia del efecto positivo que tiene sobre el desempeño, el comportamiento proactivo adoptado por las empresas inmersas en un ambiente de turbulencia económica (Ndubisi e Iftikhar, 2012; Kraus et al., 2012).

Los resultados de esos estudios indican una relación estrecha entre OE y desempeño y sugieren que su implementación no solo genera un buen desempeño; sino también, que aquellas empresas que se encuentran inmersas en un ambiente hostil, al adoptar una OE modifican la manera de cómo lograr un mejor desempeño, lo que les permite sobrellevar con mayor facilidad las dificultades impuestas por las condiciones desfavorables del contexto en que se desenvuelven, potenciando de esta forma su capacidad para sobrevivir.

La evidencia empírica proporciona la base para suponer (Diagrama 1), que en un ambiente de crisis social, económica y de la naturaleza, la OE compuesta por innovación, agresividad competitiva y proactividad, modifica la manera en que los negocios de artesanía mejoran su desempeño. 


\section{Diagrama 1 Modelo de investigación}

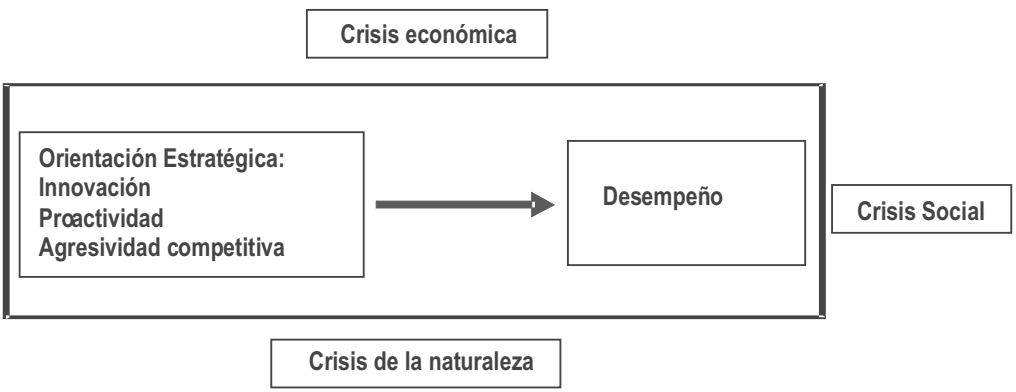

Fuente: Elaboración propia.

\section{Dimensiones de desempeño y Orientación Estratégica}

Como primer paso para evaluar el modelo de investigación se obtuvo la validez interna de los constructos mediante un análisis factorial. Los resultados obtenidos, así como la interpretación de la estructura conceptual subyacente en cada una de las variables del estudio que se detallan en secciones sub-siguientes.

- Desempeño del negocio, se midió con una medida subjetiva que fue adaptada de la escala desarrollada por Hernández et al. (2010) y Domínguez et al. (2004). Se pidió a los dueños del negocio indicar en una escala tipo Likert de cinco puntos de (1) muy insatisfecho, a (5) muy satisfecho, el grado de satisfacción con respecto a los resultados económicos del negocio y los beneficios obtenidos de la venta de artesanía; así como el grado de satisfacción personal con su actividad como artesano. En contraste con la medida objetiva del desempeño, que se basa en indicadores tales como el retorno sobre la inversión (ROI), retorno sobre capital (ROE), o el retorno sobre activos (ROA).
La medida subjetiva se utilizó por dos razones: 1) la muestra estaba compuesta principalmente por pequeños negocios, que generalmente carecen de los indicadores objetivos convencionales del desempeño utilizados por las empresas establecidas $y$, en el caso de tenerlos muestran mucho recelo para proporcionar tal información; tal como lo señala la literatura sobre las pequeñas y medianas empresas (Ingenbleek et al., 2013; Acquaah et al., 2011); 2), la muestra estaba compuesta por negocios que se dedican a la elaboración de artesanías. Estos negocios, aunque tienen por objetivo la obtención de ganancias, también le dan importancia a los aspectos intrínsecos como la satisfacción (Mayer-Haug et al., 2013; Paige y Littrell, 2002).

Además, estudios empíricos en el campo de gestión estratégica que han utilizado ambas medidas: objetivas y subjetivas, han encontrado que existe una fuerte correlación entre las dos (Dess y Robinson, 1984). Por lo tanto, en estudios como éste donde no se puede disponer de indicadores objetivos, el desempeño puede medirse con escalas subjetivas (Acquaah et al., 2011). 
Orientación estratégica y desempeño de negocios artesanales afectados por crisis... Mendoza Ramírez, Luis y Toledo López, Arcelia

El resultado del análisis factorial de la escala de desempeño mostró tres dimensiones. Con base en la interpretación de la estructura factorial subyacente en la escala, la primera dimensión se etiquetó como "satisfacción con los resultados del negocio", porque está compuesta por ítems que representan la rentabilidad del negocio. La segunda dimensión se etiquetó como "satisfacción con los beneficios del negocio", porque los ítems reflejan la rentabilidad obtenida del negocio. La tercera dimensión se etiquetó como "satisfacción personal", porque está compuesta por ítems relacionados con aspectos intrínsecos sobre la actividad artesanal. La rotación convergió en 5 iteraciones, la varianza explicada es del $80 \%$, y el alfa de Cronbach es de .92.

- Orientación estratégica, en este estudio se utilizó el enfoque multidimensional de OE. Este enfoque consistió en la utilización de tres de las cinco dimensiones que Lumpkin y Dess (1996) postulan como elementos que las empresas pueden utilizar de manera independiente, o mediante diferentes combinaciones para competir, dependiendo de sus características internas o del contexto: innovación, proactividad y agresividad competitiva. En contraste con el enfoque unidimensional (Covin y Slevin, 1989), que postula tres dimensiones -innovación, proactividad y toma de riesgos-; sobre las que la empresa debe basarse necesariamente de manera conjunta para competir.

Se utilizó el enfoque multidimensional debido a las características de los negocios de artesanía y con base en las prácticas utilizadas por estos para competir según la literatura sobre el contexto artesanal en Oaxaca. No se consideró la toma de riesgos debido a que durante las crisis, los gerentes o dueños de las empresas actúan con más prudencia y evitan riesgos. Tampoco se incluyó la autonomía porque tradicionalmente en los pequeños negocios, el dueño es el principal administrador y tomador de decisiones. Por lo tanto, en este estudio OE es la postura que adopta el negocio para competir con base en tres dimensiones: innovación, agresividad competitiva y proactividad.

- Innovación, se definió operacionalmente como el grado de cambios y/o mejoras en producto y en proceso. Esta variable se midió con una escala basada en Covin y Slevin (1989), y adaptada de Hernández et al. (2007). La escala era de tipo Likert, de cinco puntos que iba de 1: Nada, a 5: Muchísimo.

El resultado del análisis factorial de innovación mostró dos dimensiones. Con base en la interpretación de la estructura factorial subyacente en la escala, la primera dimensión se etiquetó como "mejoras en producto", y está compuesta por mejora en diseño, forma, decorado, acabado y calidad. La segunda dimensión se etiquetó como "cambios y mejoras en proceso", porque los ítems reflejan las modificaciones y el reemplazo de herramientas, maquinaria y equipo. La rotación convergió en 3 iteraciones, la varianza explicada es del $67 \%$, y el alfa de Cronbach es de .81.

- Agresividad competitiva, se definió operacionalmente como la frecuencia con que el dueño del negocio realiza acciones para mantener o aumentar la porción de mercado, aún a costa de los ingresos y las ganancias. Se midió con una escala Likert, basada en Covin y Slevin (1989), y adaptada de Escribá-Esteve 
et al. (2008) y Venkatraman (1989). La escala iba de 1: casi nunca, a 5: siempre.

El resultado del análisis factorial de agresividad competitiva mostró dos dimensiones. Con base en la interpretación de la estructura factorial subyacente en la escala, la primera dimensión se etiquetó como "precio", y está compuesta por: recorte de precios, precios competitivos y rebajas sobre venta. La segunda dimensión se etiquetó como "ganancias", y los componentes son: ventas a crédito, y pago a terceros. La rotación convergió en 3 iteraciones, la varianza explicada es del $58 \%$, y el alfa de Cronbach es de .50 .

El valor del coeficiente del alfa de Cronbach por debajo de los parámetros sugeridos de entre .70 y .90 (Celina y Campo, 2005) puede atribuirse al número reducido de ítems de la escala y a que la muestra estuvo distribuida en cuatro ramas diferentes de artesanía. Debido a que el coeficiente del alfa de Cronbach es una propiedad del patrón de respuesta de la población estudiada y no una característica de la escala en sí misma; el valor del alfa de Cronbach puede cambiar según la población en que se aplica la escala. No obstante, aunque el valor mínimo del coeficiente de alfa recomendado es de .70 , se sugiere la aceptación de valores inferiores en los casos donde no se cuenta con un mejor instrumento, pero teniendo en cuenta la baja consistencia interna de la escala utilizada (Celina y Campo, 2005).

- Proactividad, se definió operacionalmente como la actitud o predisposición del dueño del negocio hacia las acciones que tienen por objetivo derrotar a la competencia. Se midió con una escala Likert adaptada de Covin y Slevin (1989). Se pidió al encuestado su percepción sobre su similitud con los competidores con respecto a las prácticas que adoptan en el negocio para ganarle a la competencia. La escala iba de 1: no se parecen en nada, a 5: se parecen mucho.

El resultado del análisis factorial de proactividad mostró tres dimensiones. Con base en la interpretación de la estructura factorial subyacente en la escala, la primera dimensión se etiquetó como "protección de información", porque los ítems se refieren al ocultamiento de información sobre el producto, los clientes y el mercado. La segunda dimensión se etiquetó como "descrédito", porque los ítems se refieren a las acciones que desprestigian a la competencia con respecto a la calidad, la autenticidad y la accesibilidad de sus productos. La tercera dimensión se etiquetó como "tratos con terceros" y está compuesta por: tratos con agencias de viajes, guías de turistas, taxistas y otros artesanos. La rotación convergió en 5 iteraciones, la varianza explicada es del $69 \%$, y el alfa de Cronbach es de .91.

En la Tabla 1, se presentan los resultados del análisis de factores de las escalas utilizadas para medir las dimensiones que integran la OE en este estudio.

\section{Relación Orientación Estratégica-desempeño en tres ambientes de crisis}

Con la validez y fiabilidad de las variables se prosiguió con la evaluación del modelo conceptual, que plantea una relación entre dos variables -OE-desempeño- en un marco de contingencia de crisis social, económica y de la naturaleza. Por lo que se realizó un primer análisis de correlación bivariada de Pearson y luego otro de regresión múltiple, con los datos 
Orientación estratégica y desempeño de negocios artesanales afectados por crisis... Mendoza Ramírez, Luis y Toledo López, Arcelia

Tabla 1

Análisis factorial de las escalas para medir $\mathrm{OE}$

\begin{tabular}{|c|c|c|c|c|c|c|}
\hline \multirow[t]{2}{*}{ Variable } & \multirow[t]{2}{*}{ Items } & \multicolumn{3}{|c|}{ Factor } & \multirow{2}{*}{$\begin{array}{l}\text { Varianza total } \\
\text { Explicada }\end{array}$} & \multirow{2}{*}{$\begin{array}{c}\text { Alfa de } \\
\text { Cronbach }\end{array}$} \\
\hline & & 1 & 2 & 3 & & \\
\hline \multirow[t]{11}{*}{ Innovación } & Mejora en diseño & .847 & & & & \\
\hline & Mejora en forma & .823 & & & & \\
\hline & Mejora en decorado & .800 & & & & \\
\hline & Mejora en acabado & .845 & & & & \\
\hline & Mejora en calidad & .836 & & & & \\
\hline & Modificaciones a herramientas & & .790 & & & \\
\hline & Modificaciones a maquinaria & & .842 & & & \\
\hline & Reemplazo de herramientas & & .829 & & & \\
\hline & Reemplazo de equipo & & .779 & & & \\
\hline & Reemplazo de maquinaria & & .768 & & & \\
\hline & $\%$ de varianza explicada & 34.78 & 32.47 & & 67.26 & .81 \\
\hline Agresividad & Recorte & .799 & & & & \\
\hline \multirow[t]{5}{*}{ Competitiva } & Precio competitivo & .798 & & & & \\
\hline & Rebaja sobre venta & .736 & & & & \\
\hline & Ventas a crédito & & .775 & & & \\
\hline & Pago a terceros & & .663 & & & \\
\hline & $\%$ de varianza & 37.11 & 21.00 & & 58.11 & .50 \\
\hline \multirow[t]{18}{*}{ Proactividad } & Piezas que venden más & .823 & & & & \\
\hline & Diseños que gustan más & .813 & & & & \\
\hline & Diseños nuevos & .773 & & & & \\
\hline & Otros negocios de artesanía & .631 & & & & \\
\hline & Otros lugares donde se vende artesanía & .632 & & & & \\
\hline & Compradores foráneos & .825 & & & & \\
\hline & Compradores mayoristas & .867 & & & & \\
\hline & Lugares donde se vende más & .761 & & & & \\
\hline & Calidad de los productos & & .753 & & & \\
\hline & Precios de los productos & & .855 & & & \\
\hline & Origen de los productos & & .880 & & & \\
\hline & Calidad de los materiales & & .776 & & & \\
\hline & Elaboración de los productos & & .809 & & & \\
\hline & Agencia de viajes & & & .933 & & \\
\hline & Guías de turistas & & & .922 & & \\
\hline & Taxistas & & & .818 & & \\
\hline & Otros artesanos & & & .659 & & \\
\hline & $\%$ de varianza explicada & 29.97 & 21.78 & 18.05 & 69.81 & .91 \\
\hline
\end{tabular}

Análisis de componentes principales con rotación varimax con Kaiser.

Rotación convergida en 3 y 5 iteraciones.

Fuente: Elaboración propia 
sobre el desempeño del negocio en los tres ambientes de crisis.

Una vez determinada la relación entre OE y desempeño en cada uno de los ambientes de crisis. La discusión se hizo a partir del comportamiento de las dimensiones que integran cada una de las variables según el análisis factorial. De esta manera se explicó cuál es la intensidad de la relación entre las variables en cada uno de los ambientes de crisis.

\subsection{Variables de Orientación estratégica relacionadas con el desempeño del negocio}

En la Tabla 2, se muestra que en los tres ambientes de crisis existe una relación significativa entre algunas de las variables de OE y el desempeño. En concordancia con Kraus et al. (2012), que reportan una asociación positiva entre el comportamiento proactivo y el desempeño de empresas en ambientes hostiles, los resultados del presente estudio muestran que en un ambiente de crisis social, dos dimensiones de la variable proactividad se correlacionan con el desempeño: la protección de información se asocia de manera positiva con satisfacción con el negocio ( .30 , $p \leq 0.01)$ y satisfacción personal (.43, $p \leq 0.01)$. El descrédito, como dimensión de proactividad, se asocia positivamente con satisfacción personal $(.29, p \leq 0.01)$.

Los resultados revelan también, que existe una asociación negativa entre

Tabla 2

Resumen de la matriz de correlaciones

\begin{tabular}{|c|c|c|c|c|c|c|c|c|}
\hline \multirow[b]{3}{*}{$\begin{array}{l}\text { Desempeño: } \\
\text { Satisfacción }\end{array}$} & \multicolumn{8}{|c|}{ Crisis } \\
\hline & \multicolumn{2}{|c|}{ Social } & \multicolumn{3}{|c|}{ Económica } & \multicolumn{3}{|c|}{ De la naturaleza } \\
\hline & $\begin{array}{c}\text { con el } \\
\text { negocio }\end{array}$ & personal & con el $r$ & egocio & personal & con el & negocio & personal \\
\hline $\begin{array}{l}\text { Orientación } \\
\text { estratégica: }\end{array}$ & & & resultados & beneficios & & resultados & beneficios & \\
\hline $\begin{array}{l}\text { Innovación } \\
\text { en producto }\end{array}$ & .115 & .102 & .069 & .114 & .189 & $.267\left(^{* *}\right)$ & .188 & $.342(* *)$ \\
\hline $\begin{array}{l}\text { Innovación } \\
\text { en proceso }\end{array}$ & -.062 & -.090 & .144 & .054 & .143 & .188 & .023 & .148 \\
\hline $\begin{array}{l}\text { Agresividad } \\
\text { en precios }\end{array}$ & -.091 & $-.243\left(^{*}\right)$ & $-.226\left(^{*}\right)$ & $-.231\left(^{*}\right)$ & $-.244\left(^{*}\right)$ & -.043 & .026 & -.055 \\
\hline $\begin{array}{l}\text { Agresividad } \\
\text { en ganancias }\end{array}$ & .061 & -.060 & .064 & .156 & .014 & -.014 & .081 & -.116 \\
\hline $\begin{array}{l}\text { Protección } \\
\text { de información }\end{array}$ & $.307\left(^{* *}\right)$ & $.434(* *)$ & .191 & .177 & $.200\left(^{*}\right)$ & $.224\left({ }^{*}\right)$ & .005 & .163 \\
\hline Descrédito & .097 & $.296\left(^{* *}\right)$ & -.040 & .005 & .026 & -.011 & -.094 & $.247\left(^{*}\right)$ \\
\hline $\begin{array}{l}\text { Tratos con } \\
\text { terceros }\end{array}$ & -.009 & .094 & .144 & $.220\left(^{*}\right)$ & $\left..305^{* *}\right)$ & .009 & -.035 & .014 \\
\hline
\end{tabular}


Orientación estratégica y desempeño de negocios artesanales afectados por crisis... Mendoza Ramírez, Luis y Toledo López, Arcelia

agresividad competitiva en precios y satisfacción personal $(-.24, p \leq 0.05)$. Estos resultados son similares a los reportados por Lumpkin y Dess (2001), quienes encontraron que en un ambiente dinámico, agresividad competitiva se asocia negativamente con el desempeño, medido por crecimiento en ventas.

De manera similar, con soporte en estudios que reportan una relación positiva entre proactividad y desempeño (Ndubisi e Iftikhar, 2012), los resultados revelan que en un ambiente de crisis económica, la variable proactividad se relaciona de manera significativa con el desempeño mediante dos de sus dimensiones: protección de información se asocia positivamente con satisfacción personal (.20, $p \leq 0.05)$. Tratos con terceros, como dimensión de proactividad, se asocia positivamente con satisfacción con los beneficios del negocio $(.22, p \leq 0.05)$, y con satisfacción personal $(.30, p \leq 0.01)$. Los resultados muestran también, una relación negativa entre agresividad competitiva en precios y las tres dimensiones de desempeño: satisfacción con los resultados del negocio $(-.22, p \leq 0.05)$, satisfacción con los beneficios del negocio $(-.23, p \leq 0.05)$, y satisfacción personal $(-.24, p \leq 0.05)$. Estos resultados son similares a los encontrados por Lumpkin y Dess (2001), quienes reportan que en un ambiente dinámico, agresividad competitiva se asocia de manera negativa con el crecimiento en ventas como índice de desempeño.

Según los resultados, también en un ambiente de crisis de la naturaleza existe una correlación entre OE y el desempeño. Dos variables de OE se relacionan significativamente con el desempeño: Innovación y proactividad.
Innovación, mostrada por las mejoras en producto se asocia de manera positiva con satisfacción con los resultados del negocio $(.26, p \leq 0.01)$ y satisfacción personal $(.34, p \leq 0.01)$. Estos resultados son congruentes con los proporcionados por diferentes estudios, los cuales indican que en ambientes adversos, las empresas que adoptan una postura estratégica enfocada en la innovación del producto logran crecimiento (Bamiatzi y Kirchmaier, 2012; Naidoo, 2010).

Proactividad, mostrada por la protección de información se asocia de manera positiva con satisfacción con los resultados del negocio (.22, $p \leq 0.05)$. Descrédito, como dimensión de proactividad, se asocia de manera positiva con satisfacción personal $(.24, p \leq 0.05)$. Diferentes estudios proporcionan soporte a estos resultados al mostrar una asociación positiva entre el comportamiento proactivo y el desempeño de las empresas en ambientes hostiles (Kraus et al., 2012; Ndubisi e Iftikhar, 2012).

\subsection{Determinantes del desempeño del negocio}

Como se observa en la Tabla 2, en cada uno de los ambientes de crisis, algunas de las variables de OE se asocian significativamente pero de manera diferente con el desempeño. Por ello, se realizó un segundo análisis mediante cuatro modelos de regresión lineal múltiple con el fin de explicar las variables que más influyen para determinar el desempeño del negocio, en cada uno de los ambientes de crisis. En cada modelo la variable dependiente fue el desempeño y las variables independientes fueron innovación, agresividad competitiva y proactividad. 
En la Tabla 3, se muestran los resultados del análisis de regresión con los modelos que contienen las variables que explican mejor el desempeño. La interpretación de los resultados se presenta por separado para cada uno de los ambientes de crisis.

\section{- En un ambiente de crisis so-} cial, el modelo que mejor explica el desempeño del negocio es el que contiene agresividad competitiva y proactividad (modelo 3). Se encontró que en un ambiente de crisis social el desempeño del negocio es modificado por la proactividad; sin embargo la agresividad competitiva no afecta el desempeño. Según los resultados, la proactividad afecta el desempeño en un $34 \%$, pero solo lo modifica y explica en un $11 \%$.

Estos resultados indican que a pesar de lo argumentado en la literatura sobre la efectividad de la innovación (Naidoo, 2010; Hall, 1980) y la agresividad competitiva (Lumpkin y Dess, 2001), en el desempeño de las empresas en ambientes hostiles; en un ambiente de crisis social, el desempeño de los negocios está en función de un énfasis mayor en proactividad.

Esto se puede explicar porque ante la presencia de desordenes sociales, tales como el bloqueo de calles y carreteras; así como la ocurrencia constante de manifestaciones masivas; la poca afluencia de compradores a los lugares de venta de productos artesanales hace que la mejora en el producto no tenga el resultado esperado en las ventas. En contra de lo esperado, las rebajas sobre venta y el recorte en los precios van en perjuicio del desempeño. Debido a que el bajo nivel de ventas no es suficiente para recuperar cuando menos la inversión puesta en el negocio, afectando la satisfacción personal del dueño del negocio con respecto al trabajo y la vida como artesano.

En cambio, una postura enfocada en el resguardo de los productos nuevos para que no sean copiados por la competencia, y el manejo discreto de la información relacionada con el mercado; además del énfasis en el descrédito de los competidores sobre la calidad de sus productos y los materiales que utilizan en la elaboración de las artesanías; logran que el dueño del negocio perciba un mejor desempeño, manifestado por su satisfacción con las ganancias del negocio, las mejoras a la vivienda y el taller, la cobertura de las necesidades básicas y los bienes adquiridos como resultado de la venta de sus artesanías. Esa percepción de mejora en el desempeño se manifiesta también en el aumento de la satisfacción personal del artesano con respecto al reconocimiento y la valoración a su trabajo por parte de los clientes; así como con su oficio y vida cotidiana como artesano.

- En un ambiente de crisis económica, el modelo que mejor explica el desempeño del negocio es el modelo 4, que incluye innovación, agresividad competitiva y proactividad. Se encontró que en un ambiente de crisis económica el desempeño del negocio no se relaciona con la innovación, pero si es modificado por la proactividad y la agresividad competitiva; aunque agresividad competitiva lo modifica de manera negativa. Según los resultados, la agresividad competitiva y la proactividad afectan el desempeño en un $33 \%$, pero solo lo modifican y explican en un $11 \%$.

Estos resultados sugieren que el desempeño del negocio en un ambiente 
Orientación estratégica y desempeño de negocios artesanales afectados por crisis... Mendoza Ramírez, Luis y Toledo López, Arcelia

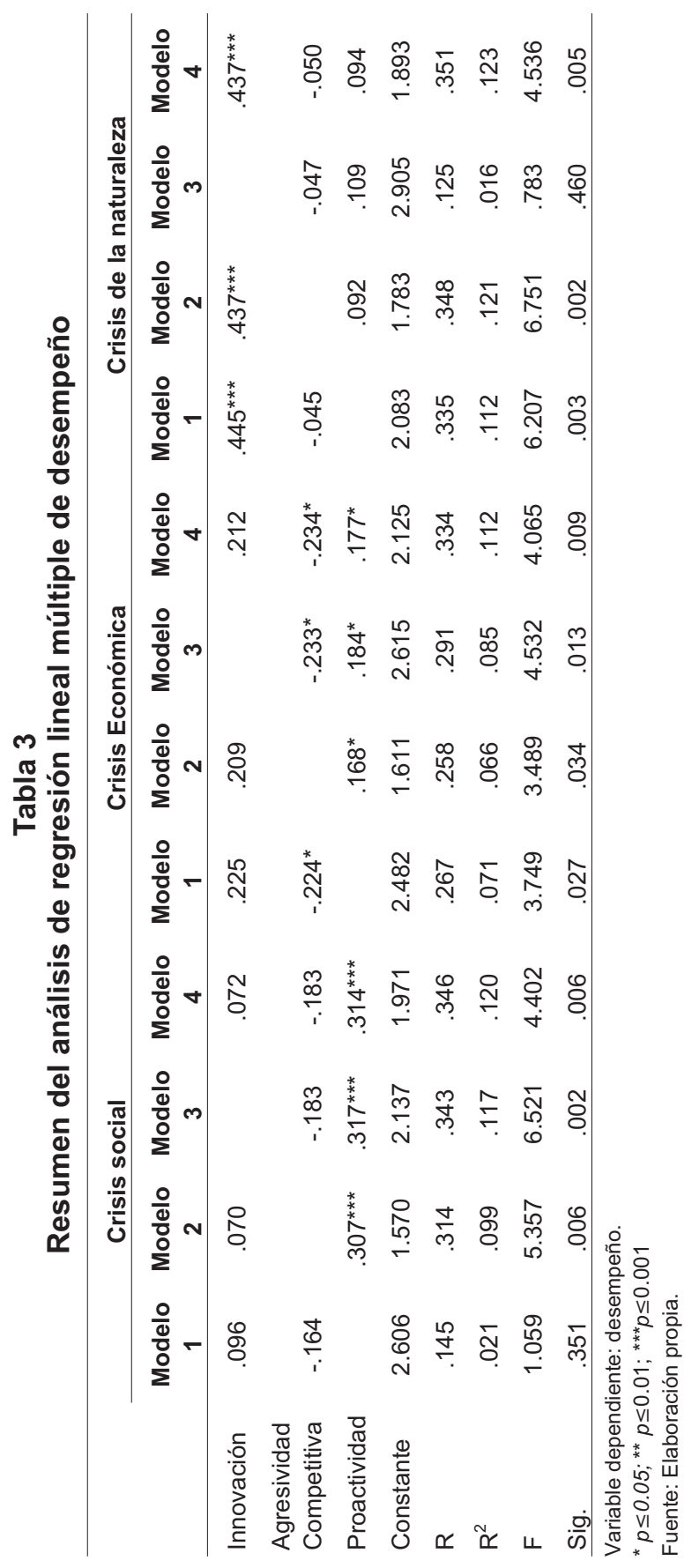


de crisis económica está determinado por la adopción de una postura proactiva; y, en contra del argumento de la supuesta efectividad de la agresividad competitiva en el desempeño de las empresas en ambientes hostiles (Lumpkin y Dess, 2001); la relación negativa significativa de la agresividad competitiva con el desempeño indica que en un ambiente de crisis económica, el desempeño del negocio está determinado por la disminución en el énfasis en el sacrificio de precios y ganancias.

Esto se puede explicar porque ante la insuficiencia de los salarios y el escaso flujo de efectivo circulante, los compradores anteponen la compra de productos básicos a los productos decorativos. Por lo que el nivel de ventas de artesanías es muy bajo, aun con la oferta de productos mejorados. Por otro lado, la adopción de una postura agresiva, mediante la oferta de precios bajos o rebajas sobre venta va en perjuicio del desempeño, debido a que los negocios de artesanía no venden siquiera lo suficiente como para recuperar la inversión puesta en el negocio. Esto genera en el dueño del negocio insatisfacción con la rentabilidad y los beneficios obtenidos por la venta de artesanías; así como insatisfacción personal con respecto al trabajo y la vida como artesano.

Mientras que el énfasis en el resguardo de los diseños nuevos y en el manejo hermético de la información sobre los gustos de los clientes y el mercado. Así como el énfasis en el establecimiento de tratos con agencias de turismo y taxistas para conseguir clientes. Esto hace que el dueño del negocio perciba una mejora en el desempeño, manifestado por la satisfacción con las mejoras a la vivienda y el taller; y los bienes adquiridos como producto de la venta de artesanía. Esa mejora en el desempeño se manifiesta también en el aumento de la satisfacción personal con respecto al cumplimiento de logros personales y el nivel de vida como artesano.

- En un ambiente de crisis de la naturaleza, el modelo que mejor explica el desempeño del negocio es el que incluye innovación y proactividad (modelo 2 ). Se encontró que el desempeño se relaciona de manera muy significativa con la innovación; sin embargo, la proactividad no afecta el desempeño. Según los resultados, la innovación afecta el desempeño en un $34 \%$, pero solo lo modifica y explica en un $12 \%$.

Estos resultados indican que en un ambiente de crisis de la naturaleza, el desempeño del negocio está en función de un fuerte énfasis en la mejora del producto y el proceso, más que en el enfoque de competencia basado en precios o la adopción de una postura proactiva.

La explicación posible a estos resultados es porque en condiciones climatológicas severas, la afluencia de compradores a los lugares de venta de productos artesanales disminuye y las materias primas para la elaboración de las artesanías escasean y suben de precio. De tal modo que, bajo estas condiciones para los negocios de artesanía no es redituable ofertar precios bajos, ni rebajas sobre venta. Debido a que con el bajo nivel de ventas apenas obtienen los ingresos suficientes para recuperar la inversión puesta en el negocio.

Por otro lado, los tratos con agencias de viajes y guías de turistas implican el desembolso de parte de las ganancias 
Orientación estratégica y desempeño de negocios artesanales afectados por crisis... Mendoza Ramírez, Luis y Toledo López, Arcelia

obtenidas de las ventas escazas; lo que resulta en el detrimento del desempeño.

Por ello, un enfoque en la mejora del producto en aspectos tales como: diseño, forma, decorado, acabado y calidad; así como en los cambios y mejoras en el proceso para adaptarlo a las condiciones climatológicas cambiantes resulta más efectivo. Debido a que con las mejoras al producto, el dueño del negocio esta en posibilidad de ofrecer una mayor variedad de mercancías y vender más piezas aunque la cantidad de clientes que llegan al negocio sea pequeña.

Además, con los cambios en el proceso se disminuyen las pérdidas de materia prima y se evitan las interrupciones en el proceso de elaboración. Esto permite al dueño del negocio la entrega de pedidos en tiempo, evitando cancelaciones y la pérdida de clientes. Estas acciones logran que el dueño del negocio perciba una mejora en el desempeño, manifestado por la satisfacción con la rentabilidad y la satisfacción personal con la reputación y el trabajo como artesano.

Aunque en un contexto diferente, los resultados de este estudio son congruentes con los resultados de estudios realizados en ambientes hostiles originados por factores diferentes a las crisis (Martins y Rialp, 2013; Rosenbusch et al., 2013; Wales et al., 2013; Kraus et al., 2012; Naidoo, 2010). Esos estudios evidencian que en ambientes hostiles, la adopción de una OE mejora el desempeño de las empresas.

Kraus et al. (2012) encuentran que en un ambiente turbulento, las empresas con OE tienen habilidades significativas para explorar y explotar las oportunidades en el ambiente; y ese comportamien- to proactivo les permite mejorar el desempeño. Martins y Rialp (2013) manifiestan que en un ambiente hostil, la $\mathrm{OE}$ juega un papel importante en el desempeño de la empresa. Según esos autores, las empresas con un perfil proactivo orientado a perseguir nuevas oportunidades y la búsqueda de información efectiva para innovar y anticiparse a las necesidades futuras del mercado, obtienen índices más altos de rentabilidad.

\section{Conclusiones}

En contra del postulado que refiere a la OE como una panacea para el desempeño de las empresas que operan en ambientes hostiles, los resultados de este estudio ponen de manifiesto la efectividad relativa que tiene la $\mathrm{OE}$ en el desempeño de pequeños negocios que operan en ambientes de crisis económica, social y de la naturaleza. Esto confirma el enfoque multidimensional de $\mathrm{OE}$; el cual argumenta que la empresa puede comprometerse en todas o en varias de sus dimensiones para mejorar el desempeño; pero no todas logran modificarlo, debido a las condiciones contextuales en que opera la empresa.

Los resultados del análisis de regresión soportan ese argumento al mostrar que no todas las variables de OE impactan en el desempeño del negocio, ni lo impactan de la misma manera en cada uno de los ambientes de crisis.

La innovación es una práctica común en los pequeños negocios de artesanía, pero en ambientes de crisis social y económica no tiene ningún efecto sobre el desempeño. La elaboración de productos innovadores y la inversión en mejoras 
del proceso generan gastos que los negocios difícilmente recuperaran con la poca afluencia de turistas en la región, provocada por la inseguridad pública, los desordenes sociales y la disminución del flujo de efectivo. Estas circunstancias provocan además, que no se puedan realizar los envíos de mercancías a los clientes, ni insertarse en otros mercados vecinos debido al cierre de carreteras, o por el riesgo de que las mercancías sean dañadas por los manifestantes populares.

En un ambiente de crisis de la naturaleza; sin embargo, el énfasis en las mejoras y los cambios, tanto en producto como en proceso, logra modificar el desempeño del negocio. No es sorprendente que en los negocios de artesanía que se comprometen con la innovación, haciendo productos con mejor diseño y acabado, y modificando los procesos para poder adaptarse a las condiciones cambiantes del clima; los dueños perciban una mejora en los resultados del negocio y una mayor satisfacción con su trabajo y estilo de vida.

Una postura proactiva enfocada en limitar las oportunidades de venta de los competidores es una estrategia generalmente utilizada por los dueños de los pequeños negocios de artesanía para competir. Sin embargo, en un ambiente de crisis de la naturaleza tal postura no tiene ningún efecto sobre el desempeño.

Bajo condiciones climáticas adversas los tratos con agencias de viajes resultan poco efectivos, debido al escaso flujo de turismo a la región. Además de que para el dueño del negocio resulta poco deseable tener que descontar de las pocas ganancias, la comisión pactada con el guía de turistas por haber llevado clientes al negocio.
La competencia basada en precios es una práctica comúnmente utilizada en los pequeños negocios de artesanía. Sin embargo, en ambientes de crisis social y de la naturaleza, esta práctica no afecta el desempeño. Ante la presencia constante de desordenes sociales y manifestaciones masivas, el flujo de turistas a la región se reduce y con ello también el nivel de ventas.

Por otro lado, bajo circunstancias climáticas adversas las materias primas se vuelven escasas y suben de precio. En este contexto, para los negocios de artesanía el ofertar precios bajos y hacer rebajas sobre venta no representa una buena opción, debido a que con el bajo nivel de ventas apenas obtienen los ingresos suficientes para recuperar la inversión en el negocio.

En un ambiente de crisis económica, en cambio, la oferta de precios bajos y las rebajas sobre venta logran modificar el desempeño, pero de manera negativa. Debido al escaso flujo de efectivo circulante y la insuficiencia de los salarios, los compradores ponen en segundo término el consumo de productos decorativos aunque sean de bajo precio. Esto repercute negativamente en el efecto que esperan los dueños de los negocios al ofertar precios más bajos, pues con las pocas ventas que realizan no recuperan ni siquiera la inversión puesta en el negocio y por el contrario, tienen pérdidas.

Este trabajo tiene importantes implicaciones para los dueños de pequeños negocios de artesanía y para los diseñadores de políticas públicas. Para los dueños de negocios artesanales, los resultados indican que en un ambiente de crisis, los negocios pueden beneficiarse al 
Orientación estratégica y desempeño de negocios artesanales afectados por crisis... Mendoza Ramírez, Luis y Toledo López, Arcelia

adoptar una OE siendo proactivos e innovadores, pero sin ser agresivos al competir en precios. Así, la OE basada en innovación y proactividad puede ser utilizada como un mecanismo para superar el reto impuesto por las condiciones desfavorables del ambiente de crisis, aprovechando las eficiencias internas del negocio y los recursos a su alcance para modificar el desempeño.

Para la comunidad que trabaja en el diseño de políticas públicas orientadas al sector artesanal, este trabajo le proporciona un apoyo para conocer qué factores son efectivos para mejorar el desempeño del negocio en un ambiente de crisis. De tal manera que, este trabajo puede servir de base para el diseño de una política encaminada al rescate de negocios de artesanía, afectados por una situación de crisis. Al indicar los elementos que se deben atender y las acciones que se deben emprender para mejorar el desempeño, considerando los medios de que disponen esos negocios, con el objetivo de lograr no solo su supervivencia, sino que vivan mejor.

\section{Referencias Bibliográficas}

Abdul-Akeem, Sadiq (2010). Digging through disaster rubble in search of the determinants of organizational mitigation and preparedness. Risk, Hazards \& Crisis in Public Policy, Volumen 1(2), United States, Texas, Berkeley Electronic Press, pp. 33-62.

Acquaah, Moses; Amoako-Gyampah, Kwasi y Jayaram, Jayanth (2011). Resilience in family and nonfamily firms: an examination of the relationships between manufacturing strategy, competitive strategy and firm performance. International journal of production re- search, Volumen 49(18), UK, Taylor \&Francis, pp. 5527-5544.

Anderson, Brian y Eshima, Yoshihiro (2011). The influence of firm age and intangible resources on the relationship between entrepreneurial orientation and firm growth among Japanese SMEs. Journal of Business Venturing, Volumen 28(3), USA, Elsevier, pp. 413429.

Bamiatzi, Vassiliki y Kirchmaier, Tom (2012). Strategies for superior performance under adverse conditions: A focus on small and medium-sized high-growth firms. International Small Business Journal, USA, SAGE Publications, pp.1-26.

Celina, Heidi y Campo, Adalberto (2005). Aproximación al uso del coeficiente alfa de Cronbach. Revista Colombiana de psiquiatría, Volumen 34(4), Colombia, Asociación Colombiana de Psiquiatría, pp. 572-580.

Chabela, Silvia (2011). Sin ventas, artesanías en Palacio de Gobierno. Noticias, voz e imagen de Oaxaca, 21-042011, Oaxaca, México, pp. 5A.

Cohen, Jeffrey (2001). Textile, tourism and community development. Annals of tourism research, Volumen 28 (2), Great Britain, Elsevier Science Ltd., pp. 378-398.

Cook, Scott (1995). B. Traven and the paradox of artisanal production in capitalism: Traven's Oaxaca tale in economic anthropological perspective. Mexican Studies/Estudios Mexicanos, Volumen 11(1), United States, University of California Press, pp. 75-111.

Covin, Jeffrey y Slevin, Dennis (1989). Strategic Management of Small Firms in Hostile and Benign Environments. Strategic Management Journal, Volumen 10(1), USA, John Wiley \& Sons, Ltd., pp. 75-87. 
Dess, Gregory y Robinson, Richard (1984). Measuring organizational performance in the absence of objective measures: The case of the privately-held firm and conglomerate business unit. Strategic Management Journal, Volumen 5(3), USA, John Wiley \& Sons, Ltd., pp. 265-273.

Domínguez, María Luisa; Hernández, José de la paz y Guzmán, Dora (2008). Orientación estratégica y desempeño en un proceso de desarrollo de productos. Alfarería en Santa María Atzompa, Oaxaca, México. Contaduría y Administración, No 225, México, Universidad Nacional Autónoma de México, pp. 79-101.

Domínguez, María Luisa; Hernández, José de la paz y Toledo, Arcelia (2004). Competitividad y ambiente en sectores fragmentados. El caso de la artesanía en México. Cuadernos de Administración, Volumen 17 (27), Colombia, Pontificia Universidad Javeriana, pp.127-158.

Elbanna, Said y Child, John (2007). The influence of decision, environmental and firm characteristics on the rationality of strategic decision-making. Journal of Management Studies, Volumen 44(4), USA, Blackwell Publishing Ltd., pp. 561-591.

Escribá-Esteve, Alejandro; Sánchez-Peinado, Luz y Sánchez-Peinado, Esther (2008). Moderating Influences on the Firm's Strategic Orientation-Performance Relationship. International Small Business Journal, Volumen 26(4), USA, SAGE Publications, pp. 463-489.

Foster, John (2010). Productivity, creative destruction and innovation policy: Some implications from the Australian experience. Innovation: Management, Policy \& Practice, Volumen 12(3), Australia, eContent Management, pp. 355-368.
Hall, William (1980). Survival strategies in a hostile environment. Harvard Business Review, September-October, USA, Harvard Business School Publishing, pp. 75-85.

Hernández, José de la paz; Domínguez, María Luisa y Caballero, Magdaleno (2005). Innovación de producto y aprendizaje dirigido en alfarería en Oaxaca, México. Revista de Ciencias Sociales, Volumen 11(2), Venezuela, Universidad del Zulia, pp. 213-228.

Hernández, José de la paz; Domínguez, María Luisa y Caballero, Magdaleno (2007). Factores de innovación en negocios de artesanía en México. Gestión y Política Pública, Volumen 16(2), México, CIDE, pp. 353-379.

Hernández, José de la paz; Domínguez, María Luisa y Mendoza, Luis (2010). Desempeño de negocios de artesanía después del movimiento social del 2006 en Oaxaca, México. Revista espiral, Volumen 16(48), México, Universidad de Guadalajara, pp.205-240.

Hua-siu, Irene (2006). The Relationship Between Entrepreneurial Orientation and Firm Performance in China. S.A.M. Advanced Management Journal, 71(3), Hong Kong, The Chinese University of Hong Kong, pp. 11-20.

Ingenbleek, Paul; Tessema, Workneh y Trijp, Hans (2013). Conducting field research in subsistence markets, with an application to market orientation in the context of Ethiopian pastoralists. International Journal of Research in Marketing, Volumen 30(1), USA, Elsevier, pp. 83-97.

Koselleck, Reinhart (2006). Crisis. Journal of the History of Ideas, Volumen 67(2), USA, Journal of the History of Ideas, pp. 357-400.

Kraus, Sascha; Rigtering, Coen; Hughes, Mathew y Hosman, Vincent (2012). Entrepreneurial orientation and the 
Orientación estratégica y desempeño de negocios artesanales afectados por crisis... Mendoza Ramírez, Luis y Toledo López, Arcelia

business performance of SMEs: a quantitative study from the Netherlands. Review of Managerial Science, Volumen 6 (2), USA, Springerlink, pp. 161-182.

Linnenluecke, Martina y Griffiths, Andrew (2010). Beyond adaptation: resilience for business in light of climate change and weather extremes. Business \& Society, Volumen 20(10), USA, SAGE Publications, pp. 1-35.

Lumpkin, George y Dess, Gregory (1996). Clarifying the entrepreneurial orientation construct and linking it to performance. Academy of Management Review, Volumen 21(1), pp.135-172.

Lumpkin, George y Dess, Gregory (2001). Linking two dimensions of entrepreneurial orientation to firm performance: the moderating rol of environment and industry life cycle. Journal of Business Venturing, Volumen 16, USA, Elsevier Science Inc., pp.429-451.

Lumpkin, George; Moss, Todd; Gras, David; Kato, Shoko y Amezcua, Alejandro (2013). Entrepreneurial processes in social contexts: how are they different, if at all? Small Business Economics, Volumen 40(3), USA, Springer, pp. 761-783.

Martínez, Víctor (2008). Crisis política y represión en Oaxaca. El cotidiano, Volumen 23(148), México, Universidad Autónoma Metropolitana-Azcapotzalco, pp. 45-62.

Martins, Izaias y Rialp, Alex (2013). Entrepreneurial orientation, environmental hostility and SME profitability: a contingency approah. Cuadernos de Gestión, Volumen 13(2), España, Instituto de economía aplicada a la empresa de la universidad del país vasco, pp. 67-88.

Mayer-Haug, Katrin; Read, Stuart; Brinckmann, Jan; Dew, Nicholas y Grichnik, Dietmar (2013). Entrepre- neurial talent and venture performance: a meta-analytic investigation of SMEs. Research policy, Obtenido el 20 de marzo de 2012 de: http://dx.doi.org/10.1016/j.respol. 2013.03.001

Mejía, Pablo (2010). Llega hoy a Oaxaca titular de la SEDESOL, evaluará daños causados por las lluvias. Obtenido el 20 de abril de 2011 de: http://www.unomasuno.com.mx/index.php?option= com_content\&view $=$ article\&id $=5647$ : llega-hoy-a-oaxaca-titular-de-la-sede sol-evaluara-danos-causados-por-la s-lluvias\&catid=116:republica\&ltemid $=515$

Munir, Kamal (2010). Financial crisis 20082009: what does the silence of institucional theorists tell us?. Journal of Management inquiry, 20(10), USA, SAGE Publications, pp. 1-4.

Naidoo, Vikash (2010). Firm survival through a crisis: the influence of market orientation, marketing innovation and business strategy. Industrial Marketing Management, Volumen 39, Australia, Elsevier, Inc, pp. 1311-1320.

Ndubisi, Nelson e Iftikhar, Khurram (2012) Relationship between entrepreneurship, innovation and performance: Comparing small and medium-size enterprises. Journal of Research in Marketing and Entrepreneurship, Volumen 14(2), USA, Emerald, pp. 214-236.

Paige, Rosalind y Litrell, Mary (2002). Craft Retailers' Criteria for Success and Associated Business Strategies. Journal of Small Business Management, Volumen 40(4), USA, John Wiley \& Sons Inc., pp. 314-331.

Rauch, Andreas; Wiklund, Johan; Lumpkin, George y Frese, Michael (2009). Entrepreneurial orientation and business performance: an assessment of past research and suggestions for the future. Entrepreneurship Theory and 
Practice, Baylor University, pp. 761787.

Ripollés, Maria y Blesa, Andreu (2006). Redes personales del empresario y orientación emprendedora en las nuevas empresas. Cuadernos de Economía y Dirección de la Empresa, No 26, España, Asociación científica de Economía y Dirección de Empresas, pp. 73-93.

Ritchie, Brent; Amaya, Carlos y Frechtling, Douglas (2010). Impacts of the world recession and economic crisis on tourism: North America. Journal of Travel Research, Volumen 49(1), USA, SAGE Publications, pp. 5-15.

Rosenbusch, Nina; Rauch, Andreas y Bausch, Andreas (2013). The Mediating Role of Entrepreneurial Orientation in the Task Environment-Performance Relationship A Meta-Analysis. Journal of Management, Volumen 39(3), USA, SAGE, pp. 633-659.

Segura, Lorena (2009). México lucha por recuperar turistas entre crisis y gripe. Obtenido el 7 de abril de 2010 de: http://lta.reuters.com/article/domesticNews/idLTASIE56M1BO200907 23 ? $\mathrm{sp}=$ true

Sorroza, Carlos (2008). La crisis política de Oaxaca: componentes, alcances y propuesta de salida. El Cotidiano, Volumen 23(148), México, UAM-Azcapotzalco, pp. 21-36.

Stephen, Lynn (2005). Women's weaving cooperatives in Oaxaca: an indigenous response to neoliberalism. Critique of anthropology, Volumen 25(3), USA, SAGE Publications, pp. 253-278.

Toledo, Arcelia; Hernández, José de la Paz y Griffin, Denis (2010). Incentives and the growth of Oaxacan subsistence business. Journal of Business Research, Volumen 63, USA, Elsevier, Inc, pp. 630-638.
Toledo-López, Arcelia; Díaz-Pichardo, René; Jiménez-Castañeda, Julio y Sánchez-Medina, Patricia (2012). Defining success in subsistence business. Journal of Business Research, Article in press, USA, Elsevier, Inc.

Venkatraman, Natarajan (1989). Strategic orientation of business enterprises: the construct, dimensionality, and measurement. Management Science, Volumen 35(8), USA, The Institute of Management Sciences, pp. 942-962.

Wales, William; Gupta, Vishal y Mousa, Farris-Terry (2013). Empirical research on entrepreneurial orientation: An assessment and suggestions for future research. International Small Business Journal, Volumen 31(4), USA, SAGE, pp. 357-383.

Wang, Yonggui y Zhang, Xiao (2009). Operationalization of corporate entrepreneurship and its performance implications in China. Journal of Chinese Entrepreneurship, Volumen 1(1), Emerald Group Publishing Limited, pp. 8-20.

Weaver, Mark; Dickson, Pat; Gibson, Brian y Turner, Andrew (2002). Being uncertain: The relationship between entrepreneurial orientation and environmental uncertainty. Journal of Enterprising Culture, Volumen 10(2), pp. 87-105.

Weick, Karl (1988). Enacted sensemaking in crisis situations. Journal of Management Studies, Volumen 25(4), USA, University of Michigan, pp. 305-317.

Zafra, Gloria (2008). Sindicalismo o educación: la paradoja del magisterio oaxaqueño. El cotidiano, Volumen 23(148), México, Universidad Autónoma Metropolitana-Azcapotzalco, pp. 139-146. 